\title{
Hypertension and consequent mortality risk in China
}

\section{Denes Stefler, PhD ${ }^{1}$, Leonardo Roever, MHS $^{2}$}

1- Department of Epidemiology and Public Health, University College London, London, UK

2- Federal University of Uberlândia, Department of Clinical Research, Uberlândia, Brazil

\section{Correspondence to:}

Denes Stefler

Department of Epidemiology and Public Health, University College London

1-19 Torrington Place, London, WC1E 6BT

Email: denes.stefler@ucl.ac.uk

Tel: 02031086281

High blood pressure is the leading cause of morbidity and mortality worldwide. In fact, according to the estimations by the Global Burden of Disease project, it was the most important risk factor among women and second among men in 2016, responsible for about 90 million and 122 million disability-adjusted life-years, respectively. ${ }^{1}$ In addition to being a 
major risk factor for coronary heart disease and stroke, as well as a key component of metabolic syndrome, it is often considered as a distinct illness on its own right. ${ }^{2,3}$

Prevalence of hypertension in Asian countries, particularly in China, is especially high. It is estimated that in this country nearly half of the adult population between the ages of 35 and 75 years has high blood pressure, with only half of these individuals are aware of their condition, less than one third are treated and less than $10 \%$ are treated adequately. ${ }^{4}$

Considering these striking numbers and the public health importance which they impose, research into the determinants, mechanism or consequences of hypertension is always valuable and relevant, particularly in this population.

In a related paper, ${ }^{5}$ Wang and colleagues examined blood pressure and blood pressure change in relation to all-cause and cause specific mortality among adults in the Yinzhou district in Eastern China.They used data from electronic health records which allowed them to include nearly 170,000 individuals in their analysis. Further advantage is that for more than 60,000 participants two waves of blood pressure measurements were available, which provided the opportunity to examine the long-term health effect of blood pressure change over a 2-year period. Conventional and standardised methods for data collection and statistical analysis were applied, and the competing risk assessment, the extensive sensitivity analyses and the propensity score adjustment added further value and strength to the work.

In terms of the results, not surprisingly, and consistently with most previous studies, ${ }^{6}$ the authors found that hypertension predicted higher all-cause and CVD mortality, while hypotension (blood pressure lower than $90 / 60 \mathrm{mmHg}$ ) also increased the risk. The findings got additional confirmation by testing the linearity of these associations in specific blood pressure groups. Clear positive link emerged only in participants with systolic or diastolic blood pressure higher than $140 \mathrm{mmHg}$ or $90 \mathrm{mmHg}$, respectively. The longitudinal analysis 
which looked at blood pressure change in relation to mortality outcomes showed similar results, suggesting high risk of CVD or all-cause death if blood pressure during the examined 2-years period changed from normotension to prehypertension or from prehypertension to hypertension.

Interestingly, the relationship between blood pressure and cancer mortality, as well as with other causes of death, was much less obvious. Only hypotensive individuals showed significantly higher riskof cancer, and the link with hypertension (and particularly with prehypertension) seem to be inverse. The available evidence regarding the blood pressurecancerassociation is rather inconsistent, and in fact, there are several studies which indicate opposite direction to the current findings. ${ }^{7,8}$ Although the authors provide a potential explanation, further exploration of the relationship between blood pressure and cancer risk is clearly needed.

The study by Wang and colleagues is not without limitations and there are several details which need to be taken into account when interpreting the results. Most of these are adequately identified and described by the authors, and here we would just emphasise the relatively short follow-up time (6 years) and the potential misclassification due to measurement bias, both of which can contribute to the above detailed findings regarding the relationship between blood pressure and cancer.

This study emphasises the importance of appropriate blood pressure control and highlights the potential consequences if it is not achieved. Considering the high prevalence of this condition in China, as well as the apparently low proportion of well-controlled cases, ${ }^{4}$ this is a particularly important message there, but relevant for any country or society. Through prevention and treatment, public health professionals and clinicians have a joint responsibility to reduce the disease burden attributable to hypertension anywhere. 
More research is needed to assess whether patients with higher levels of blood pressure can benefit from preventive measures or therapeutic interventions, especially in relation to different ethnicities and therapeutic responses, aiming for a lower global mortality rate related to altered blood pressure levels.

\section{Competing interests}

The authors declare that they have no competing interests.

\section{References}

1. GBD 2016 Risk Factors Collaborators.Global, regional, and national comparative risk assessment of 84 behavioural, environmental and occupational, and metabolic risks or clusters of risks, 1990-2016: a systematic analysis for the Global Burden of Disease Study 2016. Lancet. 2017;390:1345-1422.

2. Roever L, Resende ES, Roever-Borges AS. Hypertroponinemia, Structural Cardiac Disease, and Stroke Mortality. Stroke. 2017;48:1134-1135.

3. Roever L, Tse G, Biondi-Zoccai G. Trends in cardiovascular disease in Australia and in the world. Eur J Prev Cardiol. 2018 Jan 1:2047487318778339. doi: 10.1177/2047487318778339. [Epub ahead of print]

4. Lu J, Lu Y, Wang X et al. Prevalence, awareness, treatment, and control of hypertension in China: data from 1.7 million adults in a population-based screening study (China PEACE Million Persons Project). Lancet.2017;390:2549-2558.

5. Wang JB, Huang QC, Hu SCet al. Baseline and longitudinal change in blood pressure and mortality in a Chinese cohort. J Epidemiol Community Health. 2018 Aug 4. pii: jech2018-211050. doi: 10.1136/jech-2018-211050. [Epub ahead of print] 
6. Lacey B, Lewington S, Clarke R, et al. Age-specific association between blood pressure and vascular and non-vascular chronic diseases in 0.5 million adults in China: a prospective cohort study. Lancet Glob Health 2018;6:e641-e649.

7. Radišauskas R, Kuzmickienė I, Milinavičienė E, Everatt R.Hypertension, serum lipids and cancer risk: A review of epidemiological evidence. Medicina (Kaunas). 2016;52(2):89-98.

8. Harding JL, Sooriyakumaran M, Anstey KJ et al.Hypertension, antihypertensive treatment and cancer incidence and mortality: a pooled collaborative analysis of 12 Australian and New Zealand cohorts. J Hypertens.2016;34:149-155. 